\title{
Positron emission tomography with oxygen-15 of stunned myocardium caused by coronary artery vasospasm after recovery
}

\author{
Denis Agostini, Hidehiro Iida, Akira Takahashi
}

\begin{abstract}
Stunned myocardium is often observed after unstable angina, myocardial infarction, thrombolysis, angioplasty, and bypass surgery but rarely after coronary vasospasm. A case of stunned myocardium caused by diffuse coronary artery vasospasm and mimicking myocardial infarction is reported. The patient had an emergency coronary angiography, which showed no pathological coronary disease, but the left ventricular ejection fraction showed severe left ventricular dysfunction. Repeat coronary angiography 24 days later after medical treatment (diltiazem and nitrates) showed complete recovery of wall function, and a diffuse vasospasm was induced in both coronary arteries (left anterior and right coronary arteries). Two days later the patient underwent a positron emission tomography study with water labelled with oxygen-15 to evaluate the viable myocardium and oxygen-15 to evaluate oxidative metabolism. The results showed normal myocardial blood flow and perfusable tissue density, confirming that the myocardium was viable, and normal myocardial oxidative metabolism, reflecting the recovery of the left ventricular function.
\end{abstract}

(Br Heart f 1995;73:69-72)

Keywords: positron emission tomography, stunned myocardium, coronary artery vasospasm.

Stunned myocardium is a prolonged but temporary dysfunction of previously ischaemic myocardial tissue salvaged by coronary reperfusion after thrombolysis, percutaneous transluminal coronary angioplasty, coronary artery bypass grafting, ${ }^{1-3}$ or spontaneous vasospasm. ${ }^{34}$ Sometimes the recovery of contractility in myocardium salvaged by reperfusion is delayed for hours, days, or weeks. Stunned myocardium may mimic myocardial infarction, ${ }^{5}$ in which case revascularisation leads to the reappearance of QRS complexes and complete recovery of normal myocardial kinetics and metabolism as evaluated by positron emission tomography. ${ }^{67}$ Recently, regional oxidative metabolism has been described in humans by using gas inhalation of oxygen-15 and water labelled with oxygen-15. ${ }^{8}$ Regional myocardial blood flow and perfusable tissue density distributions permit identification of the ischaemic but still viable myocardium after an infarction. ${ }^{9}$ We report a case of stunned myocardium in a patient who had a diffuse vasospasm of the left and right coronary arteries and in whom positron emission tomography was performed to characterise the perfusion and oxidative metabolism after treatment of stunned myocardium.

\section{Case report}

A 71 year old Japanese woman was admitted to a coronary care unit with chest pain that had lasted for 15 minutes while she was at rest. She had not had any signs before the chest pain and had no risk factors for cardiovascular disease. Results of physical examination were normal, and a 12 lead electrocardiogram showed inferior $Q$ waves, with ST-T abnormalities from leads V1 to V6 and an incomplete right bundle block branch

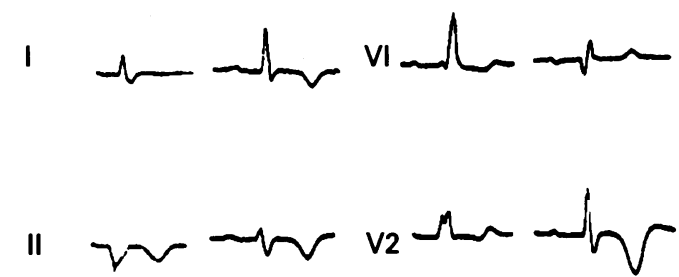



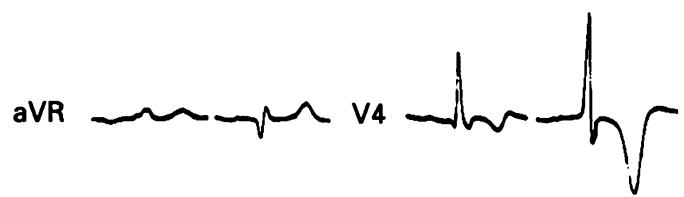

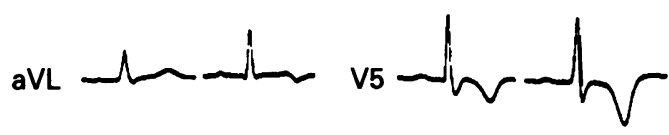

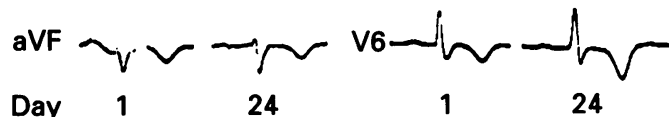

Figure 1 Electrocardiogram at admission (day 1) and 24 days later showing the transitory $Q R S$ changes that mimicked myocardial infarction. 
Figure 2 Left: At admission (day 1) angiography showed normal coronary arteries in $45^{\circ}$ left anterior oblique view of left coronary artery (LCA) and in $20^{\circ}$ cranial view of right coronary artery (RCA). Right: On day 24 intracoronary infusion of acetylcholine 10 mmolll (ACh) induced diffuse vasospasm in both right and left coronary arteries (left descending coronary artery and distal circumflex).
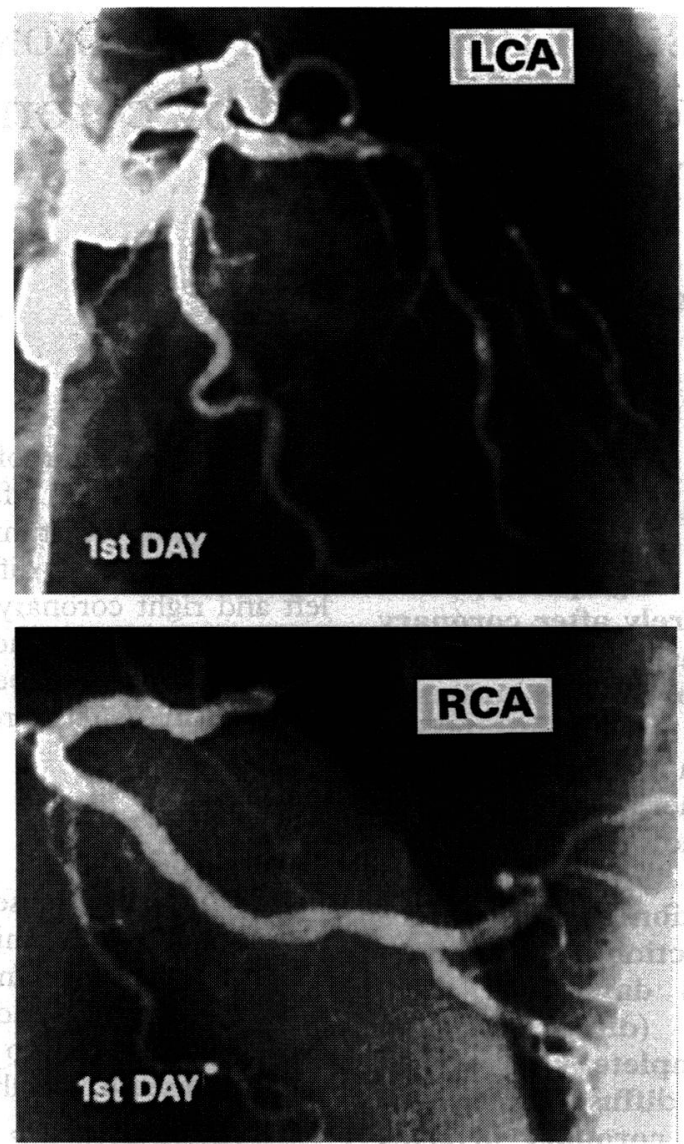

(figure 1). Cardiac enzyme concentrations (creatine kinase 58 (SD 29) IU) were normal during the three days after the onset of chest pain. Concentrations of cholesterol, triglycerides, and high and low density lipoproteins were within normal ranges. Cross sectional echocardiography showed evidence of a severe hypocontractile state along the left ventricle (left ventricular ejection fraction $28 \%$ measured by the Teischoltz method). All regional myocardial segments were evaluated qualitatively (dyskinesis, akinesis, hypokinesis, and normokinesis) and showed a diffuse hypokinesis along the left ventricle.

A consecutive series of cross sectional echocardiograms was taken over the next 24 days. The qualitative score improved gradually from diffuse hypokinesis to normokinesis, and the ejection fraction increased from $25 \%$ to $55 \%$. Owing to the unstable nature of the pain, cardiac catheterisation was performed and showed normal coronary arteries in a $45^{\circ}$ left anterior oblique view of the left coronary artery and in a $20^{\circ}$ cranial view of the right coronary artery (day 1, figure 2). The left ventriculogram showed a severe left ventricular dysfunction in a $30^{\circ}$ right anterior oblique view (left ventricular ejection fraction $35 \%$ by the Dodge method) (figure 3). The patient was treated with nitrates ( $40 \mathrm{mg}$ thrice daily) and calcium channel blockers (diltiazem 60 $\mathrm{mg}$ thrice daily) and became free of symptoms.

Ventriculography on day 24 showed a global recovery of function with a left ventricular ejection fraction of $63 \%$. A coronary
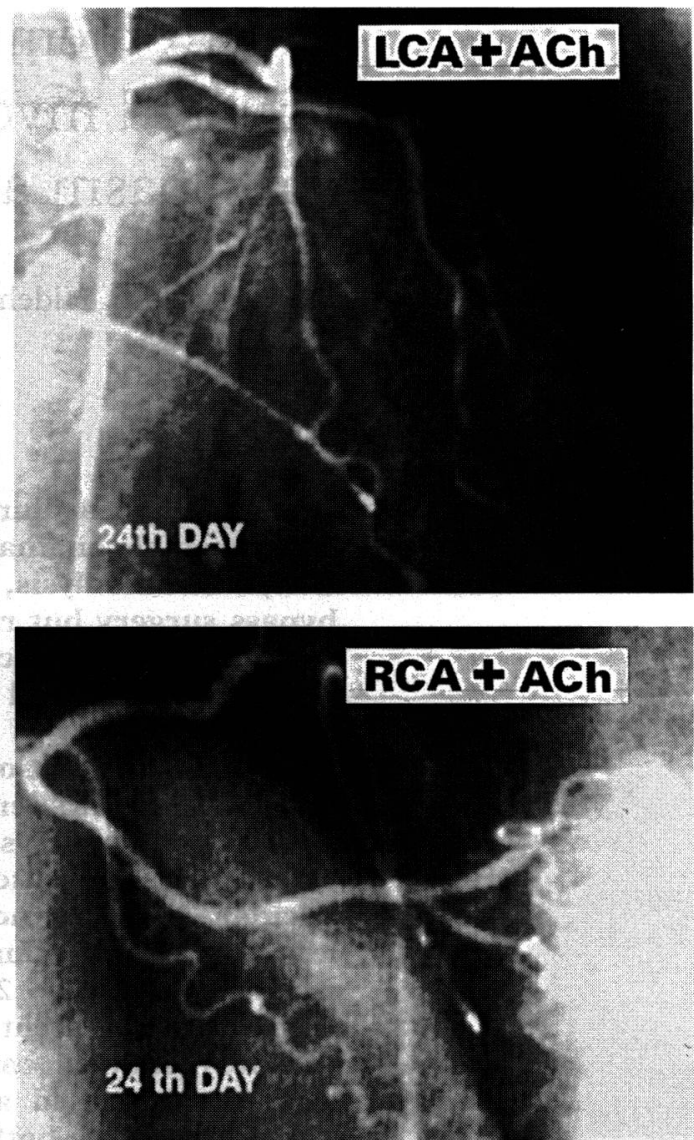

angiogram showed diffuse vasoconstriction of both coronary arteries. The left descending coronary artery and the distal circumflex artery were more vasoconstricted than the right coronary artery during the intracoronary infusion of acetylcholine $(10 \mu \mathrm{mol} / \mathrm{l})$ (figure 2). We noted a proximal left axis deviation and a diffuse irregular right coronary artery. The contrast between the electrocardiographic findings (inferior $Q$ waves) and the absence of enzymatic evidence of damaged tissue associated with the findings of both coronary arteriograms led us to assess the myocardial blood flow and oxidative metabolism by positron emission tomography.

Homogeneous perfusion (average of regional myocardial blood flow $1.10(0.5)$ $\mathrm{ml} / \mathrm{min} / \mathrm{g}$ of perfusable tissue) and perfusable tissue density (average of tissue fraction $\mathbf{0 . 6 0 6}$ $(0 \cdot 1) \mathrm{g} / \mathrm{ml})$ were found along the left ventricle, which is our criterion for viable myocardium. ${ }^{10}$ However, the distribution of oxidative metabolism corresponded to the recovery of left ventricular function (metabolic rate of oxygen $=0.121(0.07) \mathrm{ml} / \mathrm{min} / \mathrm{g}$ of perfusable tissue) (figure 4).

\section{Discussion}

Acute and severe ischaemia is followed by myocardial dysfunction, called myocardial stunning, and recovery to normal function might require a few minutes, hours, or several days. ${ }^{1-3}$ In our case the recovery to normal function was delayed compared with that of cases reported by Mathias et al and Annane 
Figure 3 Top: On day 1, lefi ventriculography showed diffuse hypokinesi with left ventricular dysfunction at end of systole $(A)$ and end diastole (B). Bottom: On day 24 angiography showed global

improvement in wall motion and recovery of lefi ventricular function in $30^{\circ}$ right anterior oblique view at end of systole (C) and end of diastole (D).


et $a .^{47}$ Brief episodes of ischaemia caused by vasospasm insufficient to produce necrosis may cause stunned myocardium and may mimic the ischaemia observed in dogs, produced by biochemical and functional changes. ${ }^{12}$

Transient electrical abnormalities and sometimes $Q$ waves may be present during episodes of vasospasm, because of transmural ischaemia. ${ }^{5}$ In our case electrocardiography showed inferior $Q$ waves, a complete subendocardial ischaemia, and an incomplete conduction abnormality. Usually, the inferior $Q$ waves are not acute, but they may indicate a severe transmural ischaemia. Electrocardio-



Figure 4 Positron emission tomograms showing midventricular transaxial slice of the myocardial blood flow (left), perfusable tissue density (middle), and myocardial oxygen uptake (right). ( $S, A, L, I$ are septal, anterior, lateral, and inferior myocardial regions, respectively; lv, left ventricle).
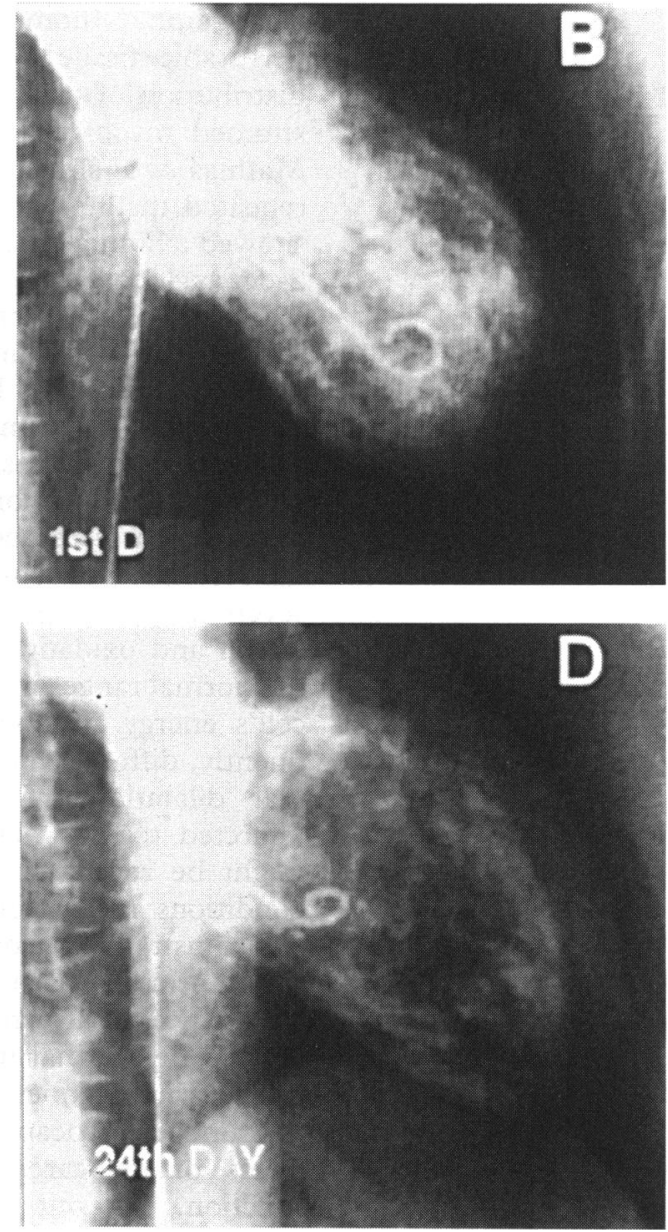

graphic changes are $T$ wave inversion, regression of inferior $\mathrm{Q}$ waves, and abnormal conduction. The giant negative $T$ waves could be due to the ischaemic event induced by acetylcholine infusion as the electrocardiogram was recorded six hours after the coronary angiogram. Although the case of stunned myocardium reported by Annane et al was not caused by vasospasm, the electrocardiogram showed a $Q$ wave in anterior leads, which became normal in two weeks. Mathias et al reported only a subendocardial injury, which recovered in 10 days. In our case first coronary angiogram and ventriculography showed severe diffuse dysfunction but no evidence of coronary artery disease. Over 24 days serial electrocardiograms and echocardiograms showed the return to normal of electrical and wall motion abnormalities. Repeated coronary angiography and ventriculography showed a complete recovery in left ventricular function and confirmed the presence of diffuse vasospasm in both coronary arteries under acetylcholine infusion. There is more evidence for diffuse vasoconstriction rather than segmental spasm, as shown in the reported cases.

Early ventriculocoronary angiography is not usually useful for decision making about prognosis and treatment, left ventricular function being generally worse than that observed a few weeks later. ${ }^{1-3}$ The lack of cardiac enzyme release, the recovery of left ventricular function, and data from positron emission 
tomography (homogeneous perfusion and perfusable tissue density and homogeneous distribution of oxidative metabolism) suggest stunned myocardium rather than infarction. Mathias et al showed myocardial viability by repeated thallium scintigraphy. ${ }^{4}$ Annane et al showed a homogeneous distribution of fluoro2-deoxyglucose, whereas perfusion was reduced in the anterior dyskinetic region when the myocardium was ischaemic but still viable. Our study is limited in understanding the recovery of stunned myocardium as we did not perform positron emission tomography when the ejection fraction was $35 \%$. Our data, which were acquired a long time after the resolution of symptoms, showed that after severe transient ischaemia the myocardial perfusion and oxidative metabolism returned to the normal range. In stunned myocardium the cell's energy metabolism is reduced; consequently, diffuse hypocontractility and ventricular dilatation may occur as they do in infarcted regions. This hypocontractile state might be reversible when normal metabolic conditions are restored. This assumption is well illustrated by our case study, in which spontaneous revascularisation and appropriate medical treatment allowed the recovery of normal myocardial metabolism and favoured full ventricular kinetic recovery.

Stunned myocardium usually recovers in terms of myocardial blood flow and wall function. In our case positron emission tomography showed normal perfusion and oxidative metabolism after treatment of stunned myocardium caused by diffuse coronary artery vasospasm.

1 Braunwald E, Kloner RA. The stunned myocardium: prolonged, postischemic ventricular dysfunction. Circulation 1982;66:1146-9.

2 Kloner RA, Przyklenk $\mathrm{K}$. Hibernation and stunning of the myocardium. N Engl ๆ Med 1992;325:1877-9.

3 Conti $C$. The stunned and hibernating myocardium: brief review. Clin Cardiol 1991;14:708-12.

4 Mathias P, Kerin NZ, Blevins RD, Cascade P, Rubenfire M. Coronary vasospasm as a cause of stunned myocardium. Am Heart $f$ 1987;113:383-5.

5 Bateman TM, Czer LSC, Gray RJ, Maddahi J, Raymond MJ, Geft II, et al. Transient pathologic Q-waves during acute ischemic events: an electrocardiographic correlate acute ischemic events: an electrocardiographic correlate of stunn 6 .

6 Brunken R, Tillisch J, Schwaiger M, Child JS, Marshall R, Mandelken $M$, et al. Regional perfusion, glucose metabolism and wall motion in patients with chronic electrocardiographic Q-wave infarctions: evidence for persistence of viable tissue in some infarct regions by positron emission tomography. Circulation 1986;73: 951-63.

7 Annane D, Py A, Duboc D, Merlet P, Fouchard J, Spaulding $\mathrm{C}$, et al. Transient left ventricular dyskinesia as a consequence of myocardial stunning. Am Heart $\mathcal{f}$ 1992;5:1377-80.

8 Iida H, Rhodes CG, Yamamoto Y, Jones T, de Silva R, Araujo LI. Quantitative measurement of myocardial metabolic rate of oxygen $\left(\mathrm{MMRO}_{2}\right)$ in man using ${ }^{15} \mathrm{O}_{2}$ and positron emission tomography [abstract]. Circulation 1990;82 (suppl III):614.

9 Iida I, Rhodes CG, de Silva R, Yamamoto Y, Araujo LI, Maseri A, et al. Myocardial tissue fraction-correction for partial volume effects and measure of tissue viability. for partial volume effects and Nucl Med 1991;32:2169-75.

10 lida H, Takahashi A, Agostini D, Tamura Y, Bloomfield PM, Ono Y. Mismatch between perfusable tissue den-
PMata sity and regional myocardial blood flow in old myocardial infarction detected by $\mathrm{H}_{2}{ }^{15} \mathrm{O}$ and PET [abstract] f Am Coll Cardiol 1993;21:461A. 